BASIC RESEARCH

\title{
New aspects of the ventricular septum and its function: an echocardiographic study
}

\author{
P Boeftler, P Claus, L Herbots, M McLaughlin, J D’hooge, B Bijnens, S Y Ho, \\ D Kececioglu, G R Sutherland
}

See end of article for authors' affiliations

Correspondence to: Dr Petra Boettler, Department of Cardiology, Catholic University of Leuven, Herestraat 49, B-3000 Leuven, Belgium; petra.boettler@email.de

Accepted 2 February 2005 Published Online First 10 March 2005

Objectives: To examine whether the line dividing the septum into two layers is found consistently by conventional echocardiography and to evaluate functional differences in the right and left side of the septum in terms of wall thickening, strain rate, and strain imaging.

Design: In a systematic study in 30 normal subjects, $M$ mode and Doppler myocardial imaging data from the interventricular septum (IVS) were recorded. Velocity curves, regional strain rate, and strain profiles were obtained. Systolic deformation (wall thickening, radial and longitudinal strain rate, and strain) of both sides were assessed. Furthermore, three patients with one sided abnormalities were studied.

Results: A bright echo consistently segmented the IVS into a left and right part. In this normal population radial deformation was different for the left and right side of the septum (mean (SD) wall thickening on the left, $49(46) \%$, and on the right, $17(38) \%$; strain rate on the left, $3.8(0.6) 1 / \mathrm{s}$, and on the right, 2.1 (1.9) $1 / \mathrm{s}$; strain on the left, $41(17) \%$, and on the right, $22(14) \%)$, whereas longitudinal deformation was found to be similar (strain rate on the left, $-2.2(0.7) 1 / \mathrm{s}$, and on the right, $-2.0(0.6) 1 / \mathrm{s}$; strain on the left, $-28(12) \%$, and on the right, -25 (12)\%). The presented clinical examples show that abnormalities can be strictly limited to one layer.

Conclusions: Differential radial deformation and knowledge of fibre architecture showing an abrupt change in the middle of the septum, together with the clinical cases, suggest the septum to be a morphologically and functionally bilayered structure potentially supplied by different coronary arteries.

$\mathrm{F}$ eigenbaum ${ }^{1}$ described a bright line within the interventricular septum (IVS) in a patient with a massively hypertrophied septum. It ran through the middle of the septum and was referred to as an "echo of unknown origin". Although in clinical practice the septum is considered to be one functional unit, with the enhanced image quality of the recent generation of echocardiographic equipment and the development of second harmonic imaging, a line with enhanced echogenicity in the IVS can often be seen.

Many investigators have shown that normal right and left ventricles do not act independently of each other and that ventricular-ventricular interaction occurs. ${ }^{23}$ The role of the septum in this interaction is still not fully determined. Some authors have suggested that ventricular-ventricular interaction is caused by the shared septal wall, ${ }^{4}$ whereas others have suggested that the free walls affect the contralateral ventricle independently of the septum. ${ }^{5}$ A model developed by Li $e^{2}$ al ${ }^{6}$ showed the impact of septal impairment on ventricular pressure development. Li et al ${ }^{6}$ showed that septal dysfunction created by glutaraldehyde injection decreased both left and right ventricular pressures. Transseptal cutting dramatically decreased left ventricular (LV) developed pressure but had no obvious influence on right ventricular pressure. Therefore, the data showed that altering septal function affected both right ventricular and LV function, but not equally. To better understand the interaction of the right sided and left sided part of the septum, Beyar et al developed a geometrical model. This model evaluated the transmural gradients in stress and strain of the left ventricle and the IVS and showed that the right and left sides of the septum respond differently to various conditions such as a one sided pressure increase by alternate aortic and pulmonary arterial constrictions. In general, there are much greater inhomogeneities in stress between the layers of the septum than between the layers of the LV free wall.
The development of tissue Doppler imaging allowed for evaluation of the differences in regional function within the septum. Fleming et $a l^{8}$ assessed total deformation of the septum by measuring the spatial distribution of the myocardial velocities between the right and left boundary of the IVS and found that there is a velocity gradient within the septum as a consequence of thickening. Kapusta et al ${ }^{9}$ found velocities within the septum to be higher in the left side than on the right side. In principle, velocity gradients can also be measured over portions of the wall, leading to an assessment of deformation at different locations. In addition, to evaluate regional differences in function of different myocardial layers newer techniques such as strain and strain rate imaging can be used to detect regional differences within the layers of the LV posterior wall. ${ }^{10}$ With the development of echocardiographic equipment and techniques, a bright line in the IVS became constantly detectable, which separates the left from the right side of the septum. Functional differences such as differences in thickening, strain, and strain rate of both sides of the septum have not been studied, though nonlinear contraction throughout the myocardium is suggested by the differing behaviours of longitudinal and circumferential fibres. ${ }^{1}$

The purpose of our study was to examine whether the bright line dividing the septum into two layers is found consistently by conventional echocardiography. We also evaluated functional differences in the right and left side of the septum by strain and strain rate imaging in a group of 30 healthy subjects. Lastly, we discuss echocardiographic findings of the septum in selected clinical cases under various pathological conditions.

Abbreviations: IVS, interventricular septum; LAD, left anterior descending artery; LV, left ventricular; LVDd, left ventricular diameter in end diastole; LVDs, left ventricular diameter in end systole 

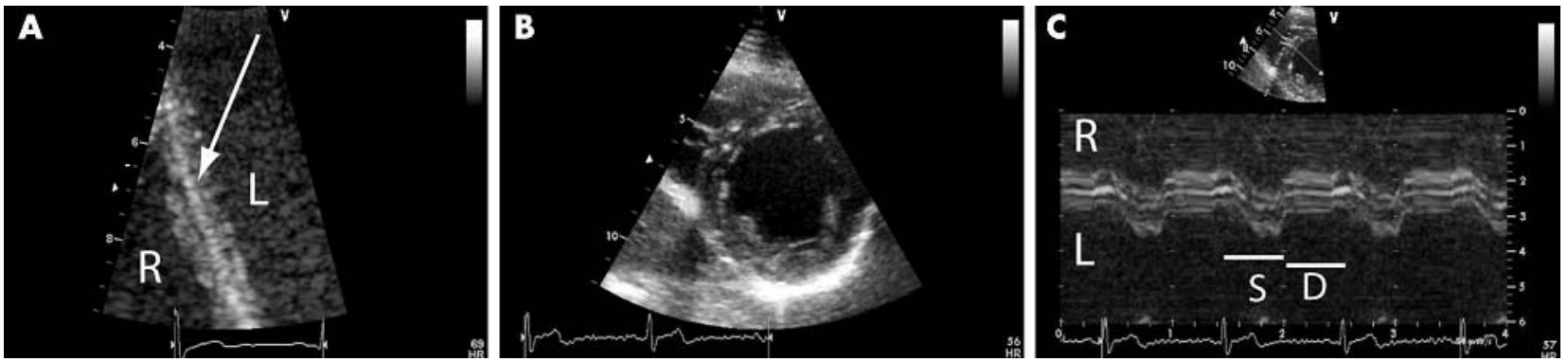

Figure 1 (A) Zoomed B mode of a normal interventricular septum in an oblique four chamber view showing the right (R) and left (L) parts of the septum separated by a bright line (arrow). (B) Parasternal short axis view. (C) Anatomical M mode of the septum in the parasternal view showing a fine line in systole (S) that brightens in diastole (D)

\section{METHODS}

\section{Study population}

The study group consisted of 30 healthy subjects (seven women and 23 men). All subjects were volunteers with no history of heart disease. Their ages ranged between 18-53 years (mean (SD) age 27.6 (8.2) years). None of the subjects had echocardiographic evidence of structural or functional heart disease. In all subjects, a 12 lead ECG was recorded, which was normal. Bicycle testing showed the maximum oxygen uptake to be within a normal range.

\section{Standard echocardiographic study}

The echocardiographic examination was performed with the subject lying in the left lateral decubitus position. Ultrasound data were acquired with a Vingmed Vivid 7 scanner (GE, Horten, Norway) and a $3.5 \mathrm{MHz}$ transducer. The images were acquired in standard and modified parasternal and apical views. Dimensions (LV diameter in end diastole (LVDd) and end systole (LVDs)), muscle thickness (IVS thickness, LV posterior wall in end diastole), and LV fractional shortening were measured in standard $\mathrm{M}$ mode grey scale. Doppler measurements with pulsed and continuous waves for all four valves were also taken.

\section{Colour Doppler myocardial imaging}

Real time two dimensional colour Doppler myocardial imaging data from the IVS were recorded in standard parasternal views at a frame rate of $>160$ frames/s. An appropriate velocity scale was chosen to avoid aliasing of colour Doppler myocardial imaging data. The narrowest image sector angle possible (usually $30^{\circ}$ ) was used to achieve the maximum colour Doppler frame rate. Three consecutive cycles were recorded during breathholding. For longitudinal deformation, Doppler myocardial imaging data of the septum were obtained in a four chamber view. Careful attention was paid to keep the ultrasound beam aligned perpendicular to the posterior wall and septum when measuring radial deformation and parallel to the septum when measuring longitudinal motion.

Table 1 Data of standard $M$ mode measurements $(n=30)$

\begin{tabular}{ll}
\hline Measurement & Mean (SD) \\
\hline IVSd $(\mathrm{cm})$ & $0.9(0.3)$ \\
LVDd $(\mathrm{cm})$ & $5.2(0.5)$ \\
LVDs $(\mathrm{cm})$ & $3.4(0.4)$ \\
LVPWd $(\mathrm{cm})$ & $0.9(0.1)$ \\
FS $(\%)$ & $35.9(5.8)$ \\
\hline
\end{tabular}

FS, fractional shortening; IVSd, interventricular septal thickness; LVDd, left ventricular diameter in end diastole; LVDs, left ventricular diameter in end systole; LVPWd, left ventricular posterior wall in end diastole.

\section{Offline analysis}

Data were stored in digital format and transferred to a computer workstation for offline analysis. This allowed the computation of thickening, strain, and strain rate by dedicated software (SPEQLE, K.U. Leuven, Leuven, Belgium).

\section{Thickness}

To quantify the radial thickening of the left and right sides of the septum, M mode clips of a standard parasternal long axis view were analysed. The borders of both sides of the septum were delineated by the bright line within the septum. Timing information was added by continuous wave or pulsed wave Doppler flow curves of the aorta to define the ejection period (from aortic valve opening to aortic valve closure). Systole was defined from the beginning of QRS to the end of ejection. End of diastole was considered to be at the onset of QRS (ECG trace). All timing information was aligned through ECG traces. The thickness was obtained at end systole and end diastole. Data were averaged over three consecutive cardiac cycles.

\section{Strain and strain rate}

From the mean velocity curves, regional strain rate was estimated from the spatial derivative of the myocardial

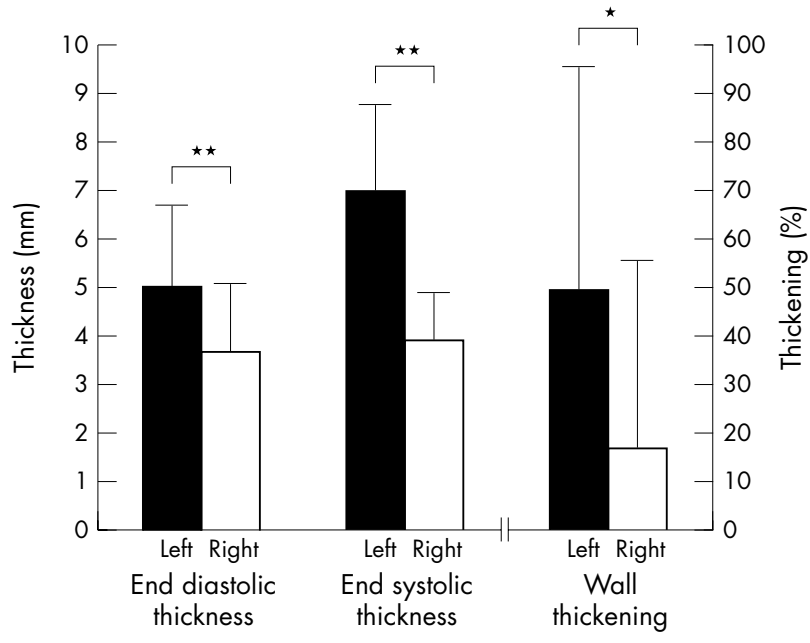

Figure 2 Septal thickness and wall thickening of the left and right sides at end diastole and end systole. ${ }^{*} p<0.002 ;{ }^{* *} p<0.0001$. 
velocity over the computation area. For strain rate, peak values during ejection were calculated. Natural strain profiles were obtained by time integrating the strain rate profile, which was averaged over three consecutive cardiac cycles, with end diastole as the reference point. Lagrangian end systolic strain values were calculated from these profiles and reported. For radial deformation, parasternal long axis views were used. The sample volumes were manually positioned in both sides of the septum. Motion was tracked throughout the cardiac cycle. To separate the right from the left side of the septum the bright line was used as a boundary. As the averaged end diastolic wall thicknesses of the right and the left sides of the septum were $3.6 \mathrm{~mm}$ (right) and $5.5 \mathrm{~mm}$ (left), the size of the computation area was fixed to $3 \mathrm{~mm}$ for radial analysis. For longitudinal deformation, strain and strain rate data were obtained from a four chamber view. The region of interest was positioned in both sides at the mid part of the septum. Strain and strain rate were calculated as described above. Computation area was fixed to $10 \mathrm{~mm}$ for longitudinal analysis. Doppler flow curves of the aortic valve and ECG traces were used for timing as described.

\section{Statistical analysis}

Values are presented as mean (SD). For comparisons of thickness, wall thickening, strain, and strain rate within both sides of the septum, a paired $t$ test was used. A probability value of $\mathrm{p}<0.05$ was considered significant.

\section{Clinical examples}

We examined three patients with one sided abnormalities such as a localised hypertrophy and myocardial infarction involving one side of the septum. Each of them underwent a full echocardiographic study, and anatomical M mode images of the septum were taken.

\section{RESULTS}

\section{Visualisation of the septum}

Standard two dimensional and $M$ mode data sets were obtained for all subjects $(\mathrm{n}=30)$. The data were uniformly of good quality and allowed subsequent offline analysis such as anatomical $\mathrm{M}$ mode. Figure $\mathrm{l}$ shows representative examples of a zoomed modified B mode of the septum and the derived $\mathrm{M}$ mode. The bright line within the septum was visualisable in all patients. This line appeared to divide the septum into a right sided and a left sided layer. The line was best and most consistently visible in the four chamber view and it was more pronounced in diastole than in systole, as fig $\mathrm{IC}$ shows. The line could be followed from the cardiac apex to the aortic root, disappearing at the left aortic cusp.

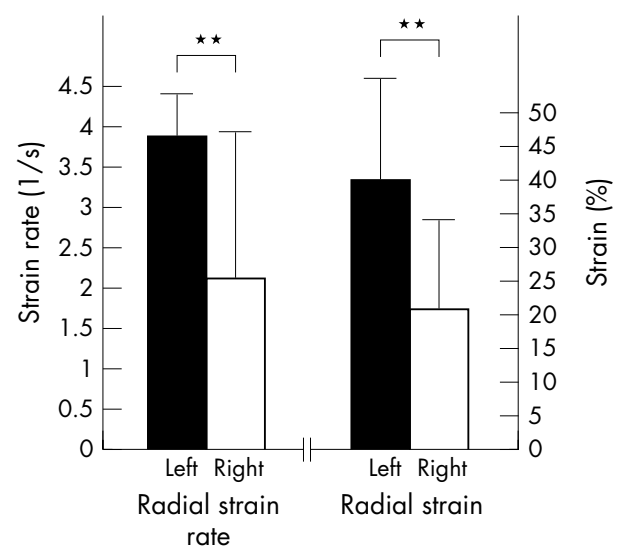

Figure 3 Peak radial strain rate and end systolic strain during ejection in the left and right sides of the septum. ${ }^{*} p<0.05 ;{ }^{* *} p<0.0001$.

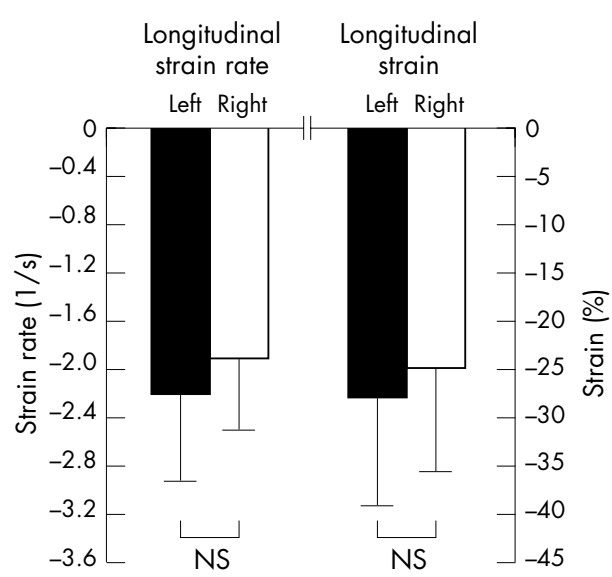

Figure 4 Peak longitudinal strain rate and end systolic strain during ejection in the left and right sides of the septum. NS, not significant.

Towards the apex it could in some patients be followed to the tip of the left ventricle.

As table 1 shows, standard $\mathrm{M}$ mode grey scale measurements of dimensions (LVDd, LVDs), muscle thickness (IVS thickness, LV posterior wall in end diastole), and LV fractional shortening were normal. Doppler measurements with pulsed and continuous waves for all four valves were also normal.

\section{Radial deformation}

Thickness

Parasternal long axis M modes for thickness evaluation were obtained for all patients. As fig 2 shows, end diastolic septal thickness was significantly less on the right side of the septum than on the left side (left, 5 (1.7) mm $v$ right, 3.6 (1.4) $\mathrm{mm}, \mathrm{p}<0.000 \mathrm{l}$ ). The thickening was predominantly located within the left side of the septum (left, $49(46) \% v$ right, $17(38) \%, \mathrm{p}<0.002)$.

\section{Strain rate and strain}

Colour Doppler myocardial imaging datasets were obtained for all subjects $(\mathrm{n}=30)$. Of 90 segments that were analysed for radial deformation, 11 were excluded because of suboptimal angles. The radial deformation of both sides of the septum was quantified in parasternal long or short axis views. Figure 3 compares data on radial strain rate and strain in the right versus the left side of the septum during ejection. Strain rate analysis showed significantly higher peak strain rates during ejection in the left side of the septum (3.8 (0.6) $\mathrm{l} / \mathrm{s}$ ) than in the right side $(2.1$ (1.9) $\mathrm{l} / \mathrm{s}, \mathrm{p}<0.05)$. In addition, the data showed significantly higher maximum strain during ejection in the left than in the right part of the septum (left, 41 (17)\% $v$ right, 22 (14)\%, p $<0.0001$ ). These values are comparable with those obtained with the $M$ mode technique (thickening). The higher standard deviation of the thickening data is due to the inherent limitation of segmenting $M$ mode data compared with quantifying Doppler images.

\section{Longitudinal deformation}

Longitudinal strain and strain rate was calculated from the Doppler myocardial imaging dataset of all patients. As fig 4 shows, peak strain rate values during ejection were similar for the left $(-2.2(0.7) \mathrm{l} / \mathrm{s})$ and right $(-2.0(0.6) \mathrm{l} / \mathrm{s})$ sides of the septum (not significant). Also, there were no significant differences in longitudinal strain between the left $(-28$ $(12) \%)$ and right $(-25(12) \%)$ sides of the septum (not significant). 

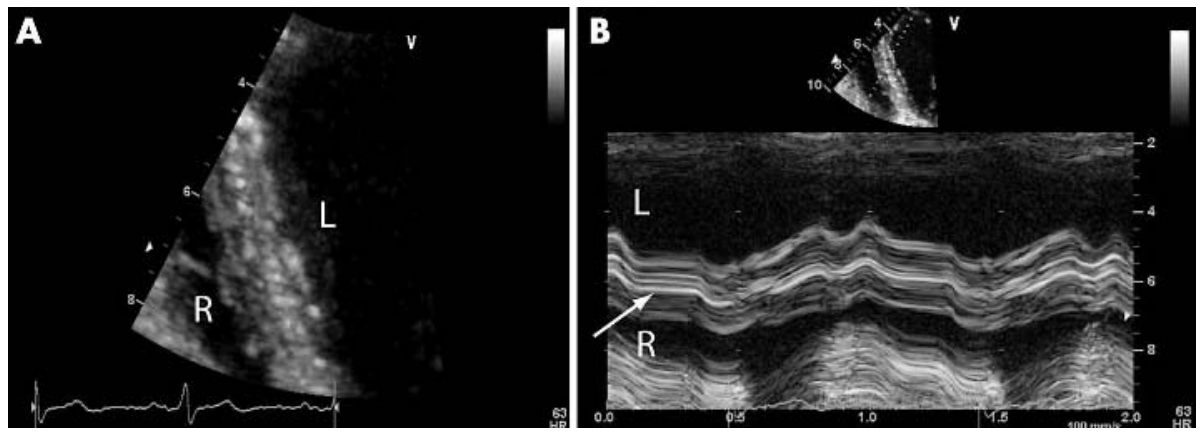

Figure 5 (A) Zoomed $B$ mode of the interventricular septum in an oblique four chamber view. (B) Anatomical M mode of image $A$ showing the moderately hypertrophied right (R) and normal left (L) part of the septum separated by a bright line of high echogenicity (arrow).

\section{Clinical examples}

Septal hypertrophy limited to the right side of the septum

A 68 year old asymptomatic man with localised nonobstructive cardiomyopathy of unknown origin was studied. The 12 lead ECG and chest radiograph were both normal. Standard grey scale and blood pool Doppler ultrasound examination showed localised hypertrophic cardiomyopathy exclusively in the right part of the septum (end diastolic wall thickness of the left septum $5 \mathrm{~mm}$, right septum $12 \mathrm{~mm}$ ). He had mild tricuspid regurgitation with a maximum gradient between the right ventricle and right atrium of $30 \mathrm{~mm} \mathrm{Hg}$ as a result of mild pulmonary hypertension. He had no pulmonary valve regurgitation. There was no evidence of LV outflow tract obstruction (peak velocity in the outflow tract $1.4 \mathrm{~m} / \mathrm{s}$ ) at rest and no mitral regurgitation. The right part of the septum was equally thickened from the apex to the base. LV dimensions (LVDd $3.4 \mathrm{~cm}$ ) and global function were normal (LV ejection fraction 59\%). As fig 5 shows, the zoomed $\mathrm{B}$ mode and its anatomical $\mathrm{M}$ mode showed a hypertrophied right part and normal left part of the septum separated by a bright line of high echogenicity.

Septal myocardial loss involving the left apical part A 58 year old man presented for a routine follow up echocardiography to our hospital. The patient's medical history was significant for a three vessel disease. Coronary angiography performed four years before had shown total occlusion of the mid right coronary artery and the mid left anterior descending artery (LAD) (various stenoses up to $100 \%$, first lateral branch totally occluded, second lateral branch $80 \%$ stenotic). The collateral circulation from the LAD to the right coronary artery through the septal branches and from the left circumflex to the LAD through the lateral branches was described and bypass surgery (left internal mammary artery to LAD) was performed. The current 12 lead ECG showed typical findings of an old anterolateral infarction-like Q wave in leads II and aVF. Chest radiography was normal. In the present echocardiography, standard grey scale and blood pool Doppler ultrasound examination showed a large myocardial infarction of the apical left part of the IVS. The infarct exclusively involved the left part of the apical septum with a normal basal septum (fig 6). The examination showed a normal right side of the septum. LV dimensions (LVDd $5.3 \mathrm{~cm}$ ) and global function were normal (LV ejection fraction $62 \%$ ).

Myocardial infarction involving the complete left sided septal layer

A 72 year old woman with a history of ischaemic heart disease presented for a routine follow up echocardiography. Coronary angiography six years previously had shown a severe stenosis (up to $90 \%$ ) of the proximal third of the LAD. In the present echocardiogram, the B mode and anatomical $M$ mode images showed a purely left sided septal myocardial infarction. The complete loss of left sided myocardium with preservation of the right side can be seen in both the B mode and the $M$ mode images (fig 7).

\section{DISCUSSION}

The anatomy and fibre structure of the free wall of the left ventricle have been examined in many studies, ${ }^{12-14}$ but surprisingly little attention has been paid to the functional morphology of the IVS. Improved image quality in echocardiography has made it possible to visualise the anatomy of the septum in detail. In this study, we have shown that a bright line between the left and right sides of the septum is found persistently in $\mathrm{B}$ and $\mathrm{M}$ mode images of conventional second harmonic echocardiography. Furthermore, we evaluated the myocardial deformation of the septum in terms of thickness, strain, and strain rate imaging and showed that the two sides differ in the radial direction but not in the longitudinal direction. Since both sides of the septum are closely connected, independent longitudinal movement is prevented and they have the same longitudinal deformation (each side influences the other). In radial motion they differ
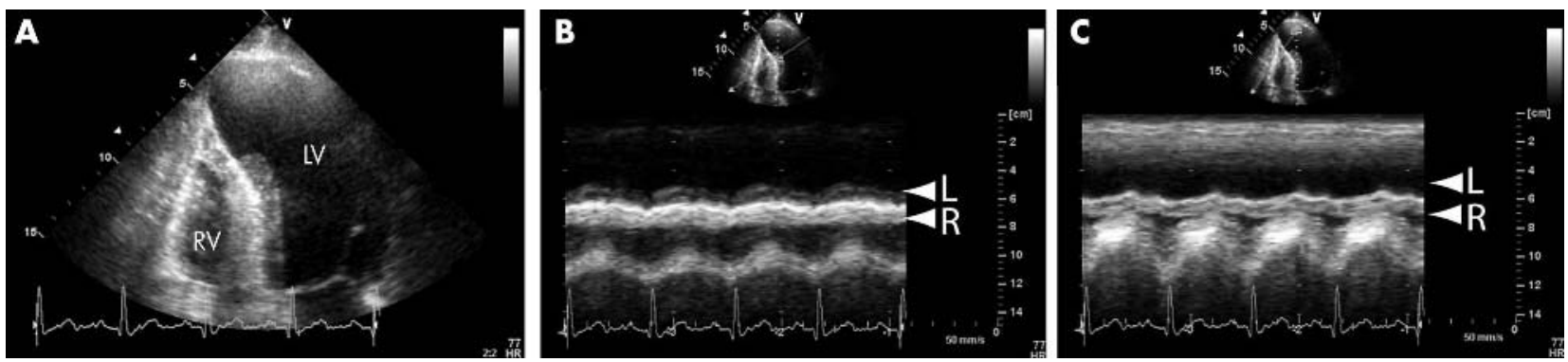

Figure 6 (A) Modified four chamber view zoomed in on the septum showing an infarcted apical part of the left sided septum. (B) Anatomical M mode through the middle part of the septum showing both sides of the septum. (C) Anatomical M mode through the apical part of the septum showing the middle line and the right (R) part of the septum with an extremely flattened left (L) part. 

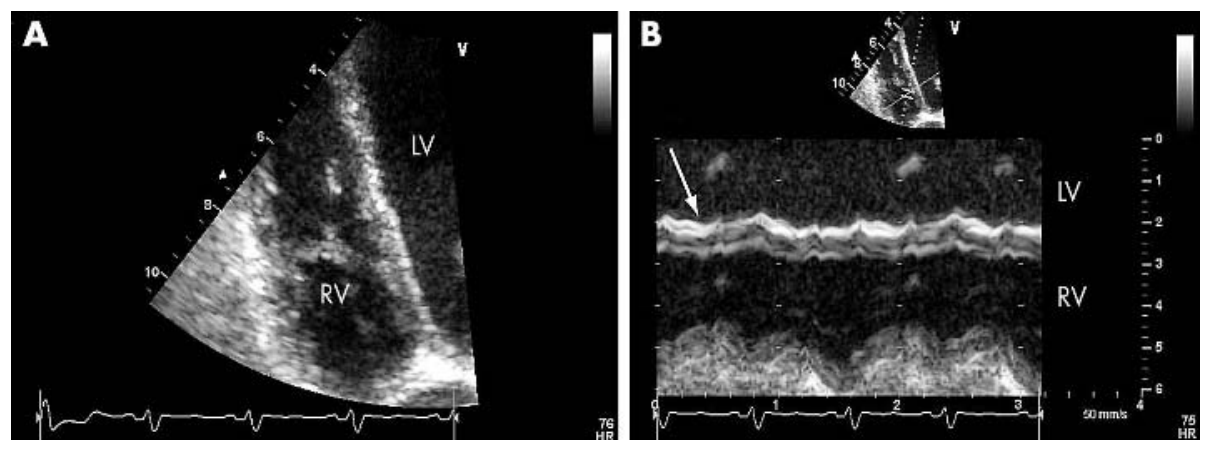

Figure 7 (A) Zoomed $B$ mode of the interventricular septum in an oblique four chamber view. (B) $M$ mode of image A showing a complete loss of myocardium on the left side of the septum (arrow).

presumably because radial expansion is oriented towards the left and right cavities and does not directly depend on the radial movement of the other side.

The newest implementations of tissue Doppler imaging have incorporated improved spatial and temporal resolutions that allow exact evaluation of regional motion. However, tethering effects of adjacent myocardium and translational heart motion can cause misinterpretation of regional wall motion. ${ }^{15-17}$ The concept of a myocardial velocity gradient and strain was developed to address these problems and enables segmental analysis of the LV independent of global heart motion. ${ }^{18-20}$ However, our analysis method requires manual tracking of the septum and LV posterior wall, which is time consuming.

The origin of the bright line separating the septum into a left and a right side is still not known. Two main hypotheses for the cause of the visualised acoustic interface are structural differences, such as an abrupt change in septal fibre direction, and the presence of coronary arteries in the middle of the septum.

Looking at the fibre orientation of the septum, there is evidence that it is a bilayered structure with an abrupt change in fibre direction. This was originally suggested by the studies of Lower from 1669. ${ }^{21}$ Fibre geometry was studied by Streeter $e a l^{13}$ and by McLean et al, ${ }^{22}$ who found very different fibre directions within two main longitudinal layers present in the IVS. Alternatively, Torrent-Guasp et al ${ }^{23}$ postulated that the heart consists of a single muscle band twisted on itself in a rope-like structure with two turns in a helical fashion. According to this theory, the septum consists of two parts, an ascending and a descending segment. However, the preparations of Torrent-Guasp et $\mathrm{al}^{23}$ might have disrupted the syncytial arrangement of the myofibres by creating a cleavage in the septum causing an artificially bilayered structure.

Additionally, several studies have shown differences in the vascular supply and the post-occlusion flow between the two sides of the septum. Farrer-Brown $e a^{24}$ found that the large septal arteries originating from the anterior and posterior descending arteries give off secondary branches that supply the right and left sides of the septum. Moreover, they describe different patterns of the secondary branches in both sides and suggest that functionally the IVS may be divided into right and left halves by the line of the main septal arteries. Ramanathan et $a l^{25}$ found significant differences in blood flow after acute septal artery occlusion in both sides of the septum. Immediately after occlusion of the septal artery, total myocardial blood flow fell dramatically within 90 seconds, with a greater decrease on the left than on the right ventricular side of the septum. This transmural asymmetry within the septal bed is directly analogous to that seen in the free wall in many studies. ${ }^{26} 27$

Overall, our findings are consistent with the septal anatomy previously described by Feigenbaum ${ }^{1}$ and the histological data of Streeter et $a l^{13}$ and Greenbaum et $a l^{12}$. The presence of the bright line may be explained by an abrupt change in fibre direction that causes an acoustic interface seen as a hyperechoic signal in the image (fig 8). We found that the line can be visualised more easily in the four chamber view than in the parasternal views. This may be due to the different acquisition angles, which may affect the imaging of interfaces. In addition, the septal coronary artery between the right and left parts of the septum as described by Farrer-Brown et al ${ }^{24}$ may contribute to the bright echogenic signal. That may also explain why the bright line is visible more clearly during diastole when coronary flow is present and coronary artery imaging should be easier.

The difference in deformation between the left and right sides that we found can be due to the structural differences as suggested above or can be due to differences in forces and geometry. Lunkenheimer et $a^{28}$ showed that there are different types of forces in the myocardium (unloading and auxotonic type) that depend on the predominant directions of the fibres.

As shown in the clinical examples, localised abnormalities may be limited to one side of the septum. For example, one side of the septum can exclusively be hypertrophic, as can be
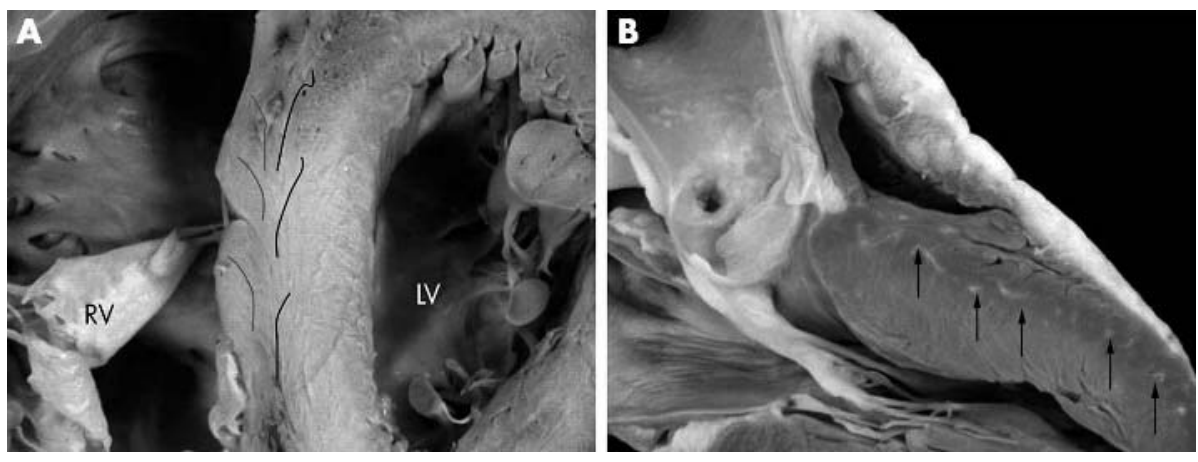

Figure 8 Specimen of the

interventricular septum showing a rapid change in fibre direction between the right side and mid layer of the septum. 
seen in a patient with localised non-obstructive cardiomyopathy (fig 5) or myocardial infarction (figs 6 and 7). These findings may be due to morphological and functional differences.

\section{Conclusion}

This study shows that the septum can be consistently divided into a left and a right side based on a bright echocardiographic signal. Also, differences in thickening and radial strain between the two sides are observable. These differences are not present in longitudinal motion. Knowledge of fibre architecture with an abrupt change in the middle of the septum, together with the discussed cases, suggests the septum to be a morphologically and functionally bilayered structure potentially supplied by different coronary arteries. Further studies to evaluate the clinical relevance of these findings are needed.

\section{ACKNOWLEDGEMENTS}

Petra Boettler was supported by a grant from the German Heart Foundation (Deutsche Herzstiftung).

\section{Authors' affiliations \\ P Boettler*, P Claus, L Herbots, M McLaughlin, J D'hooge, B Bïnens, \\ G R Sutherland, Department of Cardiology, Catholic University of Leuven, Leuven, Belgium \\ S Y Ho, Department of Paediatrics, National Heart and Lung Institute, Imperial College and Royal Brompton and Harefield NHS Trust, London, UK \\ D Kececioglu, Department of Paediatric Cardiology, Children's Hospital, Freiburg, Germany \\ *Also the Department of Paediatric Cardiology, Children's Hospital, Freiburg, Germany \\ Competing interest statement: There are no competing interests.}

\section{REFERENCES}

1 Feigenbaum H. Echocardiography, 3rd ed. Philadelphia: Lea and Febiger, 1981:454.

2 Feneley MP, Gavaghan TP, Baron DW, et al. Contribution of left ventricular contraction to the generation of right ventricular systolic pressure in the human heart. Circulation 1985;71:473-80.

3 Santamore WP, Constantinescu M, Minczak BM, et al. Contribution of each ventricular wall to ventricular interdependence. Basic Res Cardiol 1988;83:424-30.

4 Brinker JA, Weiss JL, Lappe DL, et al. Leftward septal displacement during right ventricular loading in man. Circulation 1980;61:626-33.

5 Santamore WP, Lynch PR, Heckman JL, et al. Left ventricular effects on right ventricular developed pressure. J Appl Physiol 1976;41:925-30.

6 Li KS, Santamore WP. Contribution of each wall to biventricular function. Cardiovasc Res 1993;27:792-800.
7 Beyar R, Dong SJ, Smith ER, et al. Ventricular interaction and septal deformation: a model compared with experimental data. Am J Physiol 1993;265: H2O44-56.

8 Fleming $A D, X i a X, M c D i c k e n ~ W N$, et al. Myocardial velocity gradient detected by Doppler imaging. Br J Radiol 1994;67:679-88.

9 Kapusta L, Thijssen JM, Cuypers MH, et al. Assessment of myocardial velocities in healthy children using tissue Doppler imaging. Ultrasound Med Biol 2000;26:229-37.

10 Hashimoto I, Li X, Hejmadi Bhat A, et al. Myocardial strain rate is a superior method for evaluation of left ventricular subendocardial function compared with tissue Doppler imaging. J Am Coll Cardiol 2003;42:1574-83.

11 Gibson D. Regional left ventricular wall motion. In:Roelandt JRTC, Sutherland GR, lliceto $S$, et al. Cardiac ultrasound. Edinburgh: Churchill Livingstone, 1993:241-54.

12 Greenbaum RA, Ho SY, Gibson DG, et al. Left ventricular fibre architecture in man. Br Heart J 1981;45:248-63.

13 Streeter DD, Spotnitz HM, Patel DP, et al. Fiber orientation in the canine left ventricle during diastole and systole. Circ Res 1969;24:339-47.

14 Sanchez-Quintana D, Climent V, Ho SY, et al. Myoarchitecture and connective tissue in hearts with tricuspid atresia. Heart 1999;81:182-91.

15 Urheim S, Edvardsen T, Torp H, et al. Myocardial strain by Doppler echocardiography: validation of a new method to quantify regional myocardial function. Circulation 2000;102:1158-64.

16 Abraham TP, Belohlavek $M$, Thomson $\mathrm{HL}$, et al. Time to onset of regiona relaxation: feasibility, variability and utility of a novel index of regional myocardial function by strain rate imaging. J Am Coll Cardiol 2002;39:1531-7.

17 Edvardsen T, Skulstad H, Aakhus S, et al. Regional myocardial systolic function during acute myocardial ischemia assessed by strain Doppler echocardiography. J Am Coll Cardiol 2001;37:726-30.

18 Uematsu M, Nakatani S, Yamagishi M, et al. Usefulness of myocardial velocity gradient derived from two-dimensional tissue Doppler imaging as an indicator of regional myocardial contraction independent of translational motion assessed in atrial septal defect. Am J Cardiol 1997;79:237-41.

19 Uematsu M, Miyatake K, Tanaka N, et al. Myocardial velocity gradient as a new indicator of regional left ventricular contraction: detection by a twodimensional tissue Doppler imaging technique. J Am Coll Cardiol 1995;26:217-23

20 Tsutsui $H$, Uematsu $M$, Shimizu $H$, et al. Comparative usefulness of myocardial velocity gradient in detecting ischemic myocardium by a dobutamine challenge. J Am Coll Cardiol 1998;31:89-93.

21 Lower R. Tractatus de corde: item de motu \& colore sanguinis, et chyli in eum transitu. Amsterdam: apud Danielem Elzevirium, 1669.

22 McLean M, Ross, MA, Prothero J. Three-dimensional reconstruction of the myofiber pattern in the fetal and neonatal mouse heart. Anat Rec 1989;224:392-406.

23 Torrent-Guasp F, Buckberg GD, Clemente C, et al. The structure and function of the helical heart and its buttress wrapping: the normal macroscopic structure of the heart. Semin Thorac Cardiovasc Surg 2001;13:301-19.

24 Farrer-Brown G, Rowles PM. Vascular supply of interventricular septum of human heart. Br Heart J 1969:31:727-34.

25 Ramanathan KB, Wilson JL, Mirvis DM. Effects of coronary occlusion on transmural distribution of blood flow in the interventricular septum and left ventricular free wall. Basic Res Cardiol 1988;83:229-37.

26 Becker LC, Ferreira R, Thomas M. Mapping of left ventricular blood flow with radioactive microspheres in experimental coronary artery occlusion. Cardiovasc Res 1973;7:391-400.

27 Bishop SP, White FC, Bloor CM. Regional myocardial blood flow during acute myocardial infarction in the conscious dog. Circ Res 1976;38:429-38.

28 Lunkenheimer PP, Redman K, Florek J, et al. The forces generated with the musculature of the left ventricular wall. Heart 2004;90:200-7. 